\title{
Identificação do nível de desenvolvimento regional das
microrregiões paranaenses por meio
da análise fatorial desenvolvimento regional das
microrregiões paranaenses por meio
da análise fatorial desenvolvimento regional das
microrregiões paranaenses por meio
da análise fatorial
}

Salatiel Turra ${ }^{1}$

Fernanda Mendes Bezerra Baço²

\begin{abstract}
Resumo: Este trabalho tem por objetivo identificar e analisar o desenvolvimento regional das microrregiões do estado do Paraná. Para isso, por meio da metodologia da análise fatorial é construído um nível de desenvolvimento regional, através de variáveis socioeconômicas, o qual permite examinar os fatores do desenvolvimento, bem como, classificar as microrregiões de acordo com o seu grau de desenvolvimento identificado. Os resultados mostram que, em uma escala de o a 100, o nível médio de desenvolvimento regional situou-se em 33,37. A classificação das microrregiões, através do grau de desenvolvimento, evidencia que apenas $12,82 \%$ das microrregiões do estado encontram-se na categoria de nível de desenvolvimento regional alto, tornando-se evidente que existe necessidade de focalizar políticas públicas e/ ou privadas nas microrregiões, pois a maioria delas encontra-se nas categorias de nível de desenvolvimento regional médio, baixo e muito baixo.
\end{abstract}

Palavras-chave: Análise Fatorial; Nível de Desenvolvimento Regional; Microrregiões do Paraná.

JEL: R11

1 Bacharel em Ciências Econômicas e Mestrando em Gestão e Desenvolvimento Regional (PGDR/UNIOESTE). E-mail: salatielturra@hotmail.com

2 Doutora em Economia pelo PIMES/UFPE Professora do Programa de mestrado em Gestão e Desenvolvimento Regional (PGDR/UNIOESTE). E-mail: ferpompeia@gmail.com 


\title{
Identification of regional development level of Parana's microregions through factor analysis
}

\begin{abstract}
This paper aims to identify and analyze the regional development of the micro state of Paraná. For this, using the methodology offactor analysis is built a level of regional development through socioeconomic variables, which allows us to examine the factors of development, as well as sort the micro according to their degree of development identified. The results show that, on a scale of o to 10o, the average level of regional development came to 33.37. The classification of micro, by the degree of development shows that only $12.82 \%$ of the micro state are in the category of high-level regional development, making it clear that there is need to focus public and / or private policies in the regions because most of them are in the category of medium level of regional development, low and very low.
\end{abstract}

Keywords: Factor Analysis; Level of Regional Development; Micro-regions of Paraná.

Jel: R11

\section{Introdução}

Após a crise de 1929, iniciou-se o processo de industrialização, mais precisamente, a partir da década de 1950, com o avanço tecnológico em nosso país. A introdução desta modernidade é algo bastante conflitante para os povos. Pois, como em qualquer outro aspecto e/ou conteúdo de representatividade social e econômico para a sociedade, possui seus prós e contras. De acordo com Tavares (2010), a Inglaterra e os EUA, onde o sistema capitalista era mais avançado, foram também os mais atingidos pela crise mundial de 1929. $\mathrm{E}$, em consequência disso, as disparidades de renda, inclusive no plano geográfico, foram particularmente fortes nesses países. Logo, não é casual que justamente ali tenham se dado os primeiros passos em matéria de políticas de desenvolvimento regional e urbano. Do agrupamento de contribuições surgidas na década de 1950, sem dúvida, a ideia da polarização e dos polos de desenvolvimento foi a que melhor acolheu a dinâmica territorial do desenvolvimento do capitalismo naquele contexto (Aydalot, 1985).

Dessa forma, o estudo do desenvolvimento regional deve passar por questões que vão além do aumento da situação econômica e social, proporcionadas pelo seu nível de desenvolvimento que a microrregião se classifica. O desenvolvimento regional deve ser entendido como um conceito que define a melhoria das condições financeiras e da qualidade de vida das pessoas residentes 
naquelas microrregiões.

No caso do Paraná, nas últimas décadas ocorreram importantes transformações em sua cadeia de produção agropecuária e industrial. Acentuando-se a dinamicidade da exploração econômica de ambos os setores, estabelecendo assim um novo padrão de inserção e competição para a sua economia. Neste contexto, o presente trabalho tem por objetivo identificar o desenvolvimento regional do estado do Paraná. Especificamente, pretende através de uma medida analítica, embasada pelo pressuposto econômico e social, mensurar o Nível de Desenvolvimento Regional das microrregiões geográficas do Estado por meio do método multivariado da análise fatorial e classificá-las em ordem decrescente. Para tanto, segmenta-se, além desta introdução, em três seções adicionais. Na primeira, apresenta-se o referencial teórico, em seguida a metodologia utilizada em favor da mensuração do desenvolvimento regional das microrregiões do estado do Paraná. Na seção seguinte, são apresentados os resultados observados pelas variáveis que auxiliaram, juntamente com a metodologia utilizada, na definição do nível regional, bem como, a classificação de cada uma das microrregiões em ordem decrescente. Na última seção, por fim, sistematizam - se as principais conclusões obtidas, indicando o aprofundamento de estudos para o aperfeiçoamento do assunto.

\section{Referencial Teórico}

Os conceitos de desenvolvimento regional desenvolvidos, a partir da década de 1950, chamaram atenção dos sistemas de planejamento durante os anos de 6o. Passando assim a inspirar, segundo Sorgi (2009), políticas públicas de desenvolvimento regional e estudos analíticos objetivando aferir os seus graus de adequação à realidade. Esses conceitos-chave a ser considerados são: o primeiro de pólo de crescimento, de Perroux (1955); o segundo conceito de causação circular cumulativa, de Myrdal (1957) e, o terceiro, o conceito de para trás e para frente, de Hirschman (1958). O ponto comum entre os três autores está no fato de que o crescimento ocorre de forma desiquilibrada.

Até meados dos anos 1970, o modelo de intervenção desenvolvimentista manteve-se submerso às políticas de crescimento econômico, sustentando o projeto de industrialização brasileiro. Por sua vez, serviam de auxílio as políticas de desenvolvimento regional implementadas pelas instituições federais criadas para atuar em prol de áreas menos avançadas. Segundo Uderman (2008), em 1980 as dificuldades financeiras e fiscais, juntamente com a crise do modelo desenvolvimentista influenciaram em algumas importantes mudanças no planejamento e na ação pública no Brasil. As instituições de desenvolvimento regional criadas nos anos anteriores teve sua capacidade de intervenção e formulação enfraquecidas, tornando-se órgãos incapacitados para a execução de suas atividades, as quais foram criadas. 
No início da década de 1990, o acelerado processo de abertura dos mercados e a necessidade de instituir programas de apoio à competitividade, aliados aos péssimos indicadores econômicos da década anterior, estimulou-se a retomada da ação pública em favor do desenvolvimento. Ainda, de acordo com Uderman (2008), com a estabilização monetária conquistada através da implementação do chamado Plano Real, começou a ser construído, no Brasil, um modelo de intervenção guiado por diretrizes de integração competitiva e regulação econômica, caracterizado pela adoção de políticas de cunho macroeconômico e medidas de âmbito setorial. Nesse modelo, restava pouco espaço para a formulação e a implementação de políticas de desenvolvimento regional (Udernan, 2007).

Ao tratar-se o tema "Desenvolvimento Regional", o primeiro pensamento que ocorre é de associação um modelo de sociedade, onde as microrregiões, como será abordado neste estudo, possuam alta qualidade de vida social e econômica de seus habitantes e não menos importante que isso, é identificar quais são as variáveis que favorecem tal desempenho positivo para as microrregiões com grau de desenvolvimento alto. Analisando como tendência estadual, que não se difere a nível nacional, algumas das microrregiões do Estado do Paraná atravessam por problemas como o baixo índice de ocupação de pessoas, a baixa renda, alta concentração de riquezas, a falta políticas de públicas direcionadas para a realidade das microrregiões, entre outros. $\mathrm{O}$ que se entende é que as teorias de desenvolvimento mostram não apenas os possíveis meios de alcançar os objetivos de desenvolvimento regional, mas também, as inúmeras dificuldades encontradas ao se elaborar um projeto visando ao desenvolvimento econômico/social regional.

\section{Metodologia}

\section{1 Área de estudo}

Este estudo tomará como unidade de análise as microrregiões geográficas do Estado do Paraná, com intuito de focalizar as particularidades regionais, possibilitando assim verificar desigualdades e potencialidades.

O Estado do Paraná possui como capital a cidade de Curitiba. Segundo o censo do IBGE 2010, conta com uma população de 10.444.526 habitantes. De acordo com o Ipardes (2012), possui área territorial de $199.880,200 \mathrm{~km}^{2}$, densidade demográfica de 52,92 hab/ $\mathrm{km}^{2}$ num total de 399 municípios, divididos em 10 mesorregiões e 39 microrregiões, sendo estas: Apucarana, Assaí, Astorga, Campo Mourão, Capanema, Cascavel, Cerro Azul, Cianorte, Cornélio Procópio, Curitiba, Faxinal, Floraí, Foz do Iguaçu, Francisco Beltrão, Goioerê, 
Guarapuava, Ibaiti, Irati, Ivaiporã, Jacarezinho, Jaguariaíva, Lapa, Londrina, Maringá, Palmas, Paranaguá, Paranavaí, Pato Branco, Pitanga, Ponta Grossa, Porecatu, Prudentópolis, Rio Negro, São Mateus do Sul, Telêmaco Borba, Toledo, Umuarama, União da Vitória e Wenceslau Braz.

\subsection{Método de análise}

A proposta deste estudo está baseada em trabalhos recentes que se utilizaram como sustentação de fundamentação a técnica da análise estatística multivariada, mais especificamente, a análise fatorial. O propósito em mensurar o Nível de Desenvolvimento Regional (NDR) das microrregiões seria o primeiro passo, em torno do objetivo já relatado, para em seguida classificar, de acordo com o quadro 1 e, a partir de então, identificá-las em um Ranking, de acordo com o melhor NDR.

Nesse sentido, podem-se citar inúmeros estudos que utilizam a primeira técnica. Rezende e Parré (2003) desenvolveram um trabalho embasado neste método de análise fatorial com o objetivo principal de descobrir o grau de desenvolvimento do setor agrícola dos municípios paranaenses durante a década de 1990. Nesta mesma linha rural, a dinâmica da modernização na agricultura para as 157 microrregiões homogêneas de oito estados brasileiros foi estudada em 2002 por Hoffmann, também utilizando essa mesma técnica. Aplicando duas técnicas de análise multivariada: análise fatorial e de cluster, Rezende e Parré (2004) demonstraram que o desenvolvimento da agricultura nos municípios paranaenses ocorreu de maneira diferenciada. Ainda no estado do Paraná, Melo e Parré (2007) exploraram da técnica da análise multivariada, através da análise fatorial, para mensurar o índice de desenvolvimento regional dos municípios paranaenses, apontando os determinantes que influenciaram em tal resultado dos municípios e hierarquizando-os. Para o Estado de Minas Gerais, Ferreira Júnior, Baptista e Lima (2003), realizaram um estudo acerca da modernização agropecuária nas microrregiões, através da utilização das técnicas de análise fatorial e de agrupamento. Neste mesmo Estado, Rosado, Rossato e Lima (2005) empregaram a análise fatorial, a fim de estudar a hierarquização e o desenvolvimento sócio-econômico das microrregiões. Na Bahia, através deste mesmo método apresentado, Mata et al. (2004), objetivou definir o padrão de desenvolvimento dos municípios. Foi empregada a análise fatorial por Zambrano e Pinto (2004), com a finalidade de apontar as diferenças econômicas e sociais da população dos municípios mato-grossenses. Xerxenevsky e Fochezatto (2011), no Litoral do Rio Grande do Sul, utilizou-se também este método estatístico de análise fatorial para identificar o índice relativo do desenvolvimento socioeconômico dos municípios. 


\subsection{A análise fatorial}

A análise fatorial tem como princípio básico a redução do número original de variáveis, por meio da extração de fatores independentes, de tal forma que estes fatores possam explicar, de forma simples e reduzida, as variáveis originais. Em suma, esta técnica permite extrair um número reduzido de fatores, que são combinações lineares das variáveis originais, perdendo o mínimo de informações. Este método de análise é muito empregado, como aponta Haddad (1989), para juntar regiões ou locais de acordo com a similaridade de seus perfis e agrupar variáveis para delinear padrões de variações nas características. Estes agrupamentos definem um conjunto de fatores que permitem identificar o estágio de desenvolvimento econômico, social, urbano e outros tipos de desenvolvimento de um determinado local ou região. A análise fatorial pode ser realizada através do método de componentes principais, que faz com que o primeiro fator contenha o maior percentual de explicação da variância total das variáveis da amostra, o segundo fator contenha o segundo maior percentual, e assim por diante (Ferreira Junior, Baptista e Lima, 2003).

O método de análise fatorial pode ser expresso na forma matemática através de uma combinação linear entre as variáveis $\left(\mathrm{X}_{\mathrm{i}}\right)$ e $\mathrm{K}$ fatores comuns $(\mathrm{F})$ :

$$
X_{i}=A_{i l} F_{1}+A_{i 2} F_{2}+\ldots+A_{i k} F_{k}+U_{i}+E_{i}
$$

Onde: $A_{i k}$. Cargas fatoriais, usadas para combinar linearmente os fatores comuns

$$
F_{1}, F_{2} \ldots F_{k} \text { - Fatores comuns }
$$

$U_{i}$ - Fator único

$$
E_{i} \text { - Fator de erro }
$$

As cargas fatoriais indicam a intensidade das relações entre as variáveis normalizadas Xi e os fatores. Quanto maior uma carga fatorial, mais associada com o fator se encontra a variável. A variância comum hi2, ou comunalidade, representa quanto da variância total de Xi é reproduzida pelos fatores comuns, sendo calculada a partir do somatório ao quadrado das cargas fatoriais. A variância única Ui é a parte da variância total que não se associa com a variância das outras variáveis. O termo Ei representa o erro de observação, de mensuração ou de especificação do modelo.

A medida denominada de Eingevalue ou raiz característica expressa a variância total do modelo explicada por cada fator. De acordo com Ferreira Junior, 
Baptista e Lima (2003), na determinação do número de fatores necessários para representar o conjunto de dados, usualmente consideram-se apenas os fatores cuja raiz característica é maior que a unidade. O seu valor é o somatório dos quadrados das cargas fatoriais de cada variável associadas ao fator específico. O eingevalue divido pelo número de variáveis (Xi) determina a proporção da variância total explicada pelo fator.

Para a interpretação destes fatores é realizada uma rotação ortogonal pelo método varimax, que procura minimizar o número de variáveis fortemente relacionadas com cada fator, permitindo, assim, obter fatores mais facilmente interpretáveis.

No modelo de análise fatorial, dois testes são feitos: o Kaiser-Meyer-Olkin Measure of Sampling Adequacy (KMO), que examina o ajuste dos dados, tomando todas as varáveis simultaneamente, e provê uma informação sintética sobre os dados e seu valor varia entre zero e um, sendo interpretado como adequado quando apresentar valor acima de 0,50 (Barroso e Artes, 2003). $\mathrm{O}$ outro teste, que precede a análise fatorial com vistas à verificação de suas premissas, é o Barlett Testo of Sphericity (BTS), que testa a hipótese de que a matriz de correlação é uma matriz identidade, ou seja, que não há correlação entre as variáveis.

Através da utilização do software SPSS (versão 11.5), foram obtidos os fatores e selecionados os que apresentaram valores maior que 1. Identificados os fatores, foi feita a estimação do escore fatorial, por meio do método semelhante ao da regressão. O escore para cada observação (microrregião) é resultado da multiplicação do valor (padronizado) das variáveis pelo coeficiente do escore fatorial correspondente, sendo a expressão geral para estimação do j-ésimo fator $\mathrm{Fj}$ dada por:

$$
F_{j}=W_{j 1} X_{1}+W_{j 2} X_{2}+W_{j 3} X_{3}+\ldots+W_{j p} X_{p}
$$

em que os $W_{j i}$ são os coeficientes dos escores fatoriais e $p$ é o número de variáveis.

Os escores fatoriais de cada fator possuem distribuição normal, com média zero e variância unitária e, desse modo, podem ser utilizadas para indicar a posição relativa de cada observação relativamente ao conceito expresso pelo fator. Assim, a partir da matriz dos escores fatoriais, é possível construir um índice para hierarquizar as observações (Monteiro e Pinheiro, 2004).

Através da média aritmética simples dos fatores pertencentes a cada observação (microrregião) foi obtido o Nível Bruto de Desenvolvimento Regional (NB) das microrregiões (eq. 3): 


$$
N B=\frac{\sum_{i=1}^{n}\left(F_{i}\right)}{n}
$$

Sendo:

$\mathrm{NB}$ = nível bruto (média aritmética simples dos escores fatoriais)

$F_{i}=$ escores fatoriais

$\mathrm{n}=$ número de fatores (escores fatoriais)

Tendo a finalidade de classificar as microrregiões do Estado em estudo, foi adotado o mesmo critério utilizado por Turra e Melo, (2012). Por meio da interpolação, considerou-se como o maior valor do Nível Bruto igual a 100 e o menor igual a zero, obtendo-se o Nível de Desenvolvimento Regional (NDR) para cada microrregião do Estado. O quadro 1, apresenta as categorias adotadas para classificação de desenvolvimento regional, considerando o desvio-padrão em torno da média.

QUADRO 1: CATEGORIAS DE DESENVOLVIMENTO REGIONAL

\begin{tabular}{lcr}
\hline Categoria & Sigla & \multicolumn{1}{c}{ Desvio Padrão $(\sigma)$} \\
\hline Alto & A & $A \geq(M+1 \sigma)$ \\
Médio & M & $M^{\prime}$ dia $\leq M<A$ \\
Baixo & B & $M B \leq B<$ Média \\
Muito Baixo & MB & $M B \leq(\text { Média }-1 \sigma)^{\prime}$ \\
\hline
\end{tabular}

Fonte: Elaboração própria.

Foram considerados com grau de desenvolvimento alto (A), aquelas microrregiões que obtiveram o resultado igual ou superior à soma da média mais um desvio-padrão médio (M), os que apresentam resultado no grau de desenvolvimento maior e/ou igual a média, e menor que a alta; baixo (B), aqueles com resultado da média menos um desvio-padrão, sendo apresentado como inferior à média mais superior e/ou igual ao muito baixo, e por último, os considerados muito baixo (MB), que possuem resultados no intervalo entre zero e a média menos um 1 desvio-padrão. 


\subsection{Variáveis e fontes dos dados}

O propósito de construir um Nível de Desenvolvimento Regional está intimamente ligado à análise de percepção das variáveis que influenciam as desigualdades microrregionais do território estudado. Desta maneira, mensurar o desenvolvimento regional não significa apenas expressar um indicador numérico mas também fazer comparações dos resultados das variáveis na busca da razão, a qual distingue uma da outra. O processo de desenvolvimento possui inúmeras transformações, as quais são de difícil mensuração por envolverem uma série de elementos correlacionados, sendo considerado assim um conceito complexo.

Os dados utilizados para a execução da pesquisa são secundários e obtidos através de estudos e do banco de dados do Instituto Paranaense de Desenvolvimento Econômico e Social - IPARDES e do Departamento de Economia Rural - DERAL, da Secretaria de Estado da Agricultura e do Abastecimento do Paraná - SEAB.

Existem inúmeras variáveis que retratam e definem a realidade sócio-econômico das microrregiões do estado do Paraná, através da sua mensuração. No entanto, para o segmento deste estudo foram selecionadas, conforme a tabela a seguir, 14 variáveis, as quais possuem importância relevante para mensurar o nível de desenvolvimento regional das microrregiões do Estado.

TABELA 1: IDENTIFICAÇÃO DAS VARIÁVEIS E FONTE DE DADOS

\begin{tabular}{|c|c|c|}
\hline Variáveis & Identificação das variáveis & Fonte/Ano \\
\hline $\mathrm{X} 1$ & Área territorial (Km2) & IPARDES, 2012. \\
\hline $\mathrm{X} 2$ & Densidade demográfica (hab/Km2) & IPARDES, 2012. \\
\hline $\mathrm{X}_{3}$ & Proporção da população ocupada - Total & IPARDES, 2010. \\
\hline $\mathrm{X}_{4}$ & $\begin{array}{l}\text { Valor adicionado bruto a preços básico - Total (R\$ } \\
1000,00)\end{array}$ & IPARDES, 2010. \\
\hline $\mathrm{X}_{5}$ & Valor Bruto da Produção - VBP Total $(\mathrm{R} \$ 1,00)$ & DERAL, 2012. \\
\hline $\mathrm{X} 6$ & $\begin{array}{l}\text { Fundo de Participação dos Municípios - FPM (R\$ } \\
1,00)\end{array}$ & IPARDES, 2012. \\
\hline $\mathrm{X}_{7}$ & Agências Bancárias - Total & IPARDES, 2011. \\
\hline $\mathrm{X} 8$ & PIB Per Capita (R\$1,00) & IPARDES, 2010. \\
\hline X9 & Matrículas no ensino regular - Total & IPARDES, 2012. \\
\hline $\mathrm{X} 10$ & $\begin{array}{l}\text { Matrículas na educação de jovens e adultos (EJA) - } \\
\text { Total }\end{array}$ & IPARDES, 2012. \\
\hline $\mathrm{X} 11$ & Estabelecimentos de ensino com creche - Total & IPARDES, 2011. \\
\hline $\mathrm{X} 12$ & Índice de Idosos (\%) & IPARDES, 2010. \\
\hline $\mathrm{X} 13$ & Abastecimento de água - unidades atendidas & IPARDES, 2010. \\
\hline $\mathrm{X} 14$ & Energia elétrica - Consumo (Mwh) & IPARDES, 2011. \\
\hline
\end{tabular}

Fonte: Elaboração própria. 


\section{Apresentação e Análise dos Resultados}

A análise aplicada ao modelo possibilitou a extração de três fatores com raiz característica maior que a unidade e que sintetizam as informações contidas nas 14 variáveis originais. Após rotação, conforme a Tabela 2, percebe-se que as variáveis selecionados explicam, em conjunto, $87,625 \%$ da variância total das variáveis selecionadas. O resultado desta variância é satisfatório segundo Hair et al (2009 apud Stege, 2011), pois segundo o autor, obter uma variância acumulada de $60 \%$ é satisfatório nas ciências sociais.

O teste de Bartlett ${ }^{1}$ mostrou-se significativo, rejeitando a hipótese nula de que a matriz de correlação é uma matriz identidade. O teste de KMO, para análise da adequabilidade da amostra, apresentou valor de 0,827 , indicando que a amostra é passível de ser analisada pelas técnicas da análise fatorial.

TABELA 2: RAIZ CARACTERÍSTICA, PERCENTUAL EXPLICADO POR CADA FATOR E VARIÂNCIA ACUMULADA

\begin{tabular}{lccc}
\hline Fator & Raiz característica & $\begin{array}{c}\text { Variância } \\
\text { explicada pelo } \\
\text { fator (\%) }\end{array}$ & $\begin{array}{c}\text { Variância } \\
\text { acumulada (\%) }\end{array}$ \\
\hline F1 & 8,750 & 62,501 & 62,501 \\
F2 & 1,961 & 14,006 & 76,510 \\
F3 & 1,556 & 11,115 & 87,625 \\
\hline
\end{tabular}

Fonte: Resultados da pesquisa.

A tabela 3 apresenta as cargas fatoriais e as comunalidades para os fatores considerados. Para melhor interpretação, foram consideradas apenas as cargas fatoriais com valores superiores 0,50 (destacadas em negrito), buscando evidenciar os indicadores mais fortemente associados a determinado fator. Os valores encontrados para as comunalidades revelam que praticamente todas as variáveis têm sua variabilidade captada e representada pelos três fatores.

O Fator 1 está positivo e fortemente relacionado com os indicadores X2 (Densidade demográfica (hab/ $\left.\mathrm{Km}^{2}\right), \mathrm{X}_{4}$ (Valor adicionado bruto a preços básico - Total (R\$ 1000,00), X6 (Fundo de Participação dos Municípios - FPM (R\$1,00)), X7 (Agências Bancárias - Total), X8 (PIB Per Capita (R \$ 1,00)), X9 (Matrículas no ensino regular - Total), X10 (Matrículas na educação de jovens e adultos (EJA) - Total), X11 (Estabelecimentos de ensino com creche - Total), X13 (Abastecimento de água - unidades atendidas) e X14 (Energia elétrica - Consumo (Mwh)). O Fator 1 possui a maior variância explicada pelo conjunto dos fatores, correspondendo com $62,501 \%$ do total da variância acumulada, expressando assim a importância das variáveis relacionadas para o desenvolvimento regional. Este fator está ligado à aglomeração de pessoas 1 Teste BTS: 612,622 (nível de significância: o,ooo). 
por microrregião, a economia, a educação e o bem estar das microrregiões.

TABELA 3: CARGAS FATORIAIS E COMUNALIDADES

\begin{tabular}{lcccc}
\hline \multirow{2}{*}{ Indicadores } & \multicolumn{3}{c}{ Cargas fatoriais } & $\begin{array}{c}\text { Comunali- } \\
\text { dade }\end{array}$ \\
\cline { 2 - 4 } X1 & $\mathrm{F} 1$ & $\mathrm{~F} 2$ & $\mathrm{~F} 3$ & 0,841 \\
$\mathrm{X} 2$ & 0,160 & 0,851 & $-0,301$ & 0,774 \\
X3 & 0,839 & $-0,161$ & 0,209 & 0,775 \\
X4 & 0,177 & 0,258 & 0,823 & 0,976 \\
X5 & 0,983 & 0,099 & $-0,025$ & 0,858 \\
X6 & 0,091 & 0,889 & 0,246 & 0,953 \\
X7 & 0,874 & 0,422 & 0,104 & 0,981 \\
X8 & 0,984 & 0,111 & 0,018 & 0,502 \\
X9 & 0,652 & 0,113 & $-0,254$ & 0,990 \\
X10 & 0,983 & 0,155 & $-0,028$ & 0,989 \\
X11 & 0,983 & 0,150 & $-0,012$ & 0,988 \\
X12 & 0,985 & 0,122 & 0,045 & 0,672 \\
X13 & $-0,138$ & $-0,226$ & 0,776 & 0,990 \\
X14 & 0,987 & 0,122 & 0,003 & 0,978 \\
\hline
\end{tabular}

Fonte: Resultados da pesquisa.

O fator 2 está relacionado com a área territorial (X1) e a variável X5 que expressa o Valor Bruto da Produção - VBP Total (R\$1,00). Este Fator possui a segunda maior variância explicada pelo conjunto de fatores, correspondendo a 14,006\% do total da variância explicada pelo fator. Justifica-se a ocorrência deste fato nos dias atuais, principalmente naquelas regiões onde se faz presente o uso da tecnologia para o aumento da produtividade de produtos agrícolas. Porém, deve-se levar em consideração o espaço territorial, pois como estamos analisando todas as microrregiões do estado do Paraná, é interessante destacar que existe carência de tecnologia em determinados municípios, o que pode, por um lado, diminuir a produtividade, mais aumentar a área de produção explorada. É possível associar este fator a representatividade do setor rural para a microrregião.

O fator 3 está positivo e fortemente relacionado com as variáveis X3 (Proporção da população ocupada - Total) e a X12 (índice de idosos). Tal correlação pode estar associada ao fato de que, as microrregiões onde apresentam o índice de idoso elevado, possui forte tendência em apresentar uma proporção da população ocupada inferior, o que causa consequências econômicas inesperadas, as quais influenciam na classificação e no Ranking das microrregiões, de acordo com o NDR. Por outro lado, as microrregiões com um maior índice de idosos, pode estar associada a expectativa de vida elevada que tal microrregião 
tem proporcionado as pessoas desta faixa etária. Este fator está relacionado ao desenvolvimento da renda e da qualidade de vida.

Algo bastante importante a ser considerado nas cargas fatoriais destacadas, conforme a tabela anterior, é que nenhuma delas apresentou sinal negativo, passivelmente de ser considerado que nenhuma das variáveis, conforme o fator de maior relevância, tem relação inversa na formulação do nível de desenvolvimento regional.

O passo seguinte, após a verificação das cargas fatoriais, é observar os escores fatoriais, ou seja, o valor do fator para cada município. A análise do fator deve ser feita levando em conta que seus escores originais, quando considerados todas as microrregiões em conjunto, são variáveis com média zero. Portanto, pode-se interpretar que os escores com valores próximos de zero indicam nível de desenvolvimento médio e, quanto maior em relação a zero for o fator, mais avançado será o município, no que se refere ao significado do fator em consideração.

Após a interpolação dos resultados do Índice Bruto, numa escala de zero a 100, o Nível de Desenvolvimento Regional (NDR) médio situou-se em 33,37; o desvio-padrão foi de 20,90 gerando os seguintes limites inferiores e superiores para a determinação da categoria do grau de desenvolvimento, conforme Tabela 4 .

TABELA 4: INTERVALOS DO NDR E GRAUS DE DESENVOLVIMENTO REGIONAL

\begin{tabular}{ccc}
\hline Categoria & Limite Inferior & Limite Superior \\
\hline A & 54,28 & 100,00 \\
M & 33,37 & 54,27 \\
B & 12,47 & 33,36 \\
MB & 0,00 & 12,46 \\
\hline
\end{tabular}

Fonte: Resultados da pesquisa.

A tabela 5 apresenta os resultados para as microrregiões do estado do Paraná. Verifica-se que apenas cinco microrregiões do estado se concentram no maior Nível de Desenvolvimento Regional (alto, A), totalizando 12,82\% do total das microrregiões. Já a proporção das microrregiões que se concentram na categoria de nível de desenvolvimento regional médio foi na ordem de 30,77\%. Assim, a maioria se situa na categoria de baixo e muito baixo nível de desenvolvimento regional, respectivamente, $(46,15 \%)$ e (10,26\%).

Na tabela 5, é possível observar os valores dos Fatores (F1, F2 e F3), o Nível Bruto, o Nível de Desenvolvimento Regional - NDR, o Grau de Desenvolvimento - GD e o Ranking da microrregião no Estado. 
TABELA 5: FATORES, NÍVEL BRUTO, NÍVEL DE DESENVOLVIMENTO REGIONAL, GRAU DE DESENVOLVIMENTO E RANKING DAS MICRORREGIÕES DO PARANÁ

\begin{tabular}{|c|c|c|c|c|c|c|c|}
\hline MICRORREGIÕES & $\begin{array}{c}\text { FATOR } \\
\text { o1 }\end{array}$ & $\begin{array}{c}\text { FATOR } \\
02\end{array}$ & $\begin{array}{c}\text { FATOR } \\
\text { 03 }\end{array}$ & $\begin{array}{l}\text { NÍVEL } \\
\text { BRUTO }\end{array}$ & NDR & GD & Ranking \\
\hline Curitiba & 5,693 & 0,113 & $-0,2847$ & 1,84 & 100 & $\mathrm{~A}$ & 1 \\
\hline Toledo & $-0,102$ & 2,952 & 1,4205 & 1,423 & 84,9 & $\mathrm{~A}$ & 2 \\
\hline Francisco Beltrão & $-0,269$ & 1,502 & 1,1931 & 0,809 & 62,7 & A & 3 \\
\hline Cascavel & 0,084 & 1,927 & 0,2283 & 0,747 & 60,4 & A & 4 \\
\hline Umuarama & $-0,187$ & 1,18 & 0,7403 & 0,578 & 54,3 & $\mathrm{~A}$ & 5 \\
\hline Paranavaí & $-0,17$ & 1,294 & 0,3418 & 0,488 & 51,1 & $\mathrm{M}$ & 6 \\
\hline Londrina & 1,046 & $-0,581$ & 0,8243 & 0,43 & 48,9 & M & 7 \\
\hline Astorga & $-0,173$ & 0,332 & 0,9929 & 0,384 & 47,3 & $\mathrm{M}$ & 8 \\
\hline Guarapuava & $-0,228$ & 2,587 & $-1,3141$ & 0,348 & 46 & $\mathrm{M}$ & 9 \\
\hline Maringá & 1,104 & $-1,502$ & 1,2561 & 0,286 & 43,7 & M & 10 \\
\hline Capanema & $-0,328$ & $-0,368$ & 1,4623 & 0,255 & 42,6 & $\mathrm{M}$ & 11 \\
\hline Apucarana & 0,21 & $-0,542$ & 0,9682 & 0,212 & 41 & $\mathrm{M}$ & 12 \\
\hline Cianorte & $-0,218$ & $-0,085$ & 0,7935 & 0,164 & 39,3 & $\mathrm{M}$ & 13 \\
\hline Campo Mourão & $-0,172$ & 0,532 & o,0315 & 0,13 & 38,1 & M & 14 \\
\hline Ivaiporã & $-0,356$ & 0,065 & o,6535 & 0,121 & 37,7 & $\mathrm{M}$ & 15 \\
\hline Pato Branco & $-0,131$ & 0,074 & 0,4162 & 0,12 & 37,7 & M & 16 \\
\hline Foz do Iguaçú & 0,346 & 0,427 & $-0,6889$ & 0,028 & 34,4 & M & 17 \\
\hline Prudentópolis & $-0,468$ & 0,367 & $-0,0372$ & $-0,046$ & 31,7 & B & 18 \\
\hline Cornélio Procópio & $-0,182$ & $-0,319$ & 0,3478 & $-0,051$ & 31,5 & B & 19 \\
\hline Goioerê & $-0,298$ & $-0,142$ & 0,2774 & $-0,054$ & 31,4 & B & 20 \\
\hline Floraí & $-0,332$ & $-1,23$ & 1,3295 & $-0,078$ & 30,6 & B & 21 \\
\hline Wenceslau Braz & $-0,359$ & $-0,416$ & 0,4899 & $-0,095$ & 29,9 & B & 22 \\
\hline Faxinal & $-0,398$ & $-0,987$ & 1,0997 & $-0,095$ & 29,9 & B & 23 \\
\hline Ponta grossa & 0,253 & 0,476 & $-1,2573$ & $-0,176$ & 27 & B & 24 \\
\hline Porecatu & $-0,284$ & $-0,957$ & 0,51 & $-0,244$ & 24,6 & B & 25 \\
\hline Pitanga & $-0,462$ & $-0,175$ & $-0,1186$ & $-0,252$ & 24,3 & B & 26 \\
\hline Irati & $-0,33$ & $-0,637$ & 0,0983 & $-0,289$ & 22,9 & B & 27 \\
\hline Jacarezinho & $-0,212$ & $-0,742$ & 0,0014 & $-0,317$ & 21,9 & B & 28 \\
\hline União da Vitória & $-0,348$ & $-0,076$ & $-0,8057$ & $-0,41$ & 18,5 & B & 29 \\
\hline São Mateus do Sul & $-0,377$ & $-0,655$ & $-0,3528$ & $-0,462$ & 16,7 & $\mathrm{~B}$ & 30 \\
\hline Telêmaco Borba & $-0,345$ & 0,717 & $-1,7953$ & $-0,474$ & 16,2 & B & 31 \\
\hline Ibaiti & $-0,408$ & $-0,77$ & $-0,2622$ & $-0,48$ & 16 & B & 32 \\
\hline Rio negro & $-0,253$ & $-0,627$ & $-0,5874$ & $-0,489$ & 15,7 & B & 33 \\
\hline Assaí & $-0,334$ & $-1,113$ & $-0,0263$ & $-0,491$ & 15,6 & B & 34 \\
\hline Lapa & $-0,326$ & $-0,809$ & $-0,5877$ & $-0,574$ & 12,6 & $\mathrm{~B}$ & 35 \\
\hline
\end{tabular}




\begin{tabular}{lccccccc}
\hline MICRORREGIÕES & $\begin{array}{c}\text { FATOR } \\
\text { O1 }\end{array}$ & $\begin{array}{c}\text { FATOR } \\
\text { O2 }\end{array}$ & $\begin{array}{c}\text { FATOR } \\
\text { O3 }\end{array}$ & $\begin{array}{r}\text { NÍVEL } \\
\text { BRUTO }\end{array}$ & NDR & GD & Ranking \\
\hline Palmas & $-0,325$ & $-0,232$ & $-1,5293$ & $-0,695$ & 8,2 & MB & 36 \\
Paranaguá & 0,325 & $-0,478$ & $-2,1434$ & $-0,765$ & 5,66 & MB & 37 \\
Jaguariaíva & $-0,251$ & $-0,261$ & $-2,195$ & $-0,902$ & 0,7 & MB & 38 \\
Cerro Azul & $-0,437$ & $-0,838$ & $-1,4902$ & $-0,922$ & 0 & MB & 39 \\
\hline
\end{tabular}

Fonte: Resultados da pesquisa.

Por meio dos escores fatoriais de cada microrregião, é possível verificar os elementos que mais contribuíram para o seu desenvolvimento regional. Observa-se que as cinco regiões com os maiores NDRs, ou seja, com nível de desenvolvimento regional alto (A), possuem os melhores resultados do nível bruto (NB), o qual retrata o resumo das variáveis analisadas. O que difere no entanto, é apenas a intensidade (peso característico) que cada fator possui com determinada microrregião.

Neste contexto, torna-se considerável observar que das microrregiões que estão classificadas no melhor Ranking, com exceção de Curitiba, por tornar-se atípica das demais devido a sua situação de ser uma microrregião metropolitana, as outras possuem o Fator 2 mais elevado significativamente, ou seja, o setor rural possui influência acentuada para o desenvolvimento regional destas microrregiões. A líder deste Ranking tem relação forte com o Fator 1, o qual expressa o grau de aglomeração de pessoas, as melhores condições da economia, educação e do bem estar da microrregião.

No outro extremo, pode-se observar que as microrregiões com NDR muito baixo (MB) tiveram todos os fatores (Fator 1, Fator 2 e Fator 3) negativos, refletindo os aspectos que carecem mais atenção, ao considerar as variáveis com maior correlação com tais fatores. As interpretações atribuídas anteriormente ao F3, está exemplificada claramente na análise, que é possível ser feita, das microrregiões de NDR muito baixo. Pois, 100\% delas, ou seja, as últimas quatro classificadas no Ranking, possuem maior correlação das variáveis analisadas com o F3. Comprovando-se, portanto, que quanto maior o índice de idoso e menor a proporção da população ocupada, o desenvolvimento regional em termos econômicos daquelas regiões serão menores.

Dando continuidade a esta analise, podemos perceber que através dos dados apresentados, cabe atrair a atenção a quantidade de microrregiões que possuem NDRs igual ou inferior à Média $(\mathrm{M}),(\mathrm{NDR}$ Médio $=33,37)$, pois 34 microrregiões apresentam-se com nível M, B e MB de desenvolvimento regional. Vale destacar que a microrregião de Cerro Azul, a qual possui NDR igual a o,oo (zero), não significa que a mesma não seja desenvolvida, uma vez que a mesma está classificada nesta ordem de acordo com as variáveis selecionadas, a metodologia utilizada e o grupo de microrregiões analisadas, sendo passível outro estudo com o mesmo objetivo, através de variáveis di- 
ferentes, que resultem em outra ordem de classificação.

Após serem calculados os NDRs e classificadas pelo Grau de Desenvolvimento as microrregiões, é possível observar, através da Figura 1, como o NDR está distribuído espacialmente e de forma heterogênea no Estado.

FIGURA 1: DISTRIBUIÇÃO ESPACIAL DO NÍVEL DE DESENVOLVIMENTO REGIONAL DAS MICRORREGIÕES GEOGRÁFICAS DO ESTADO DO PARANÁ, 2013.

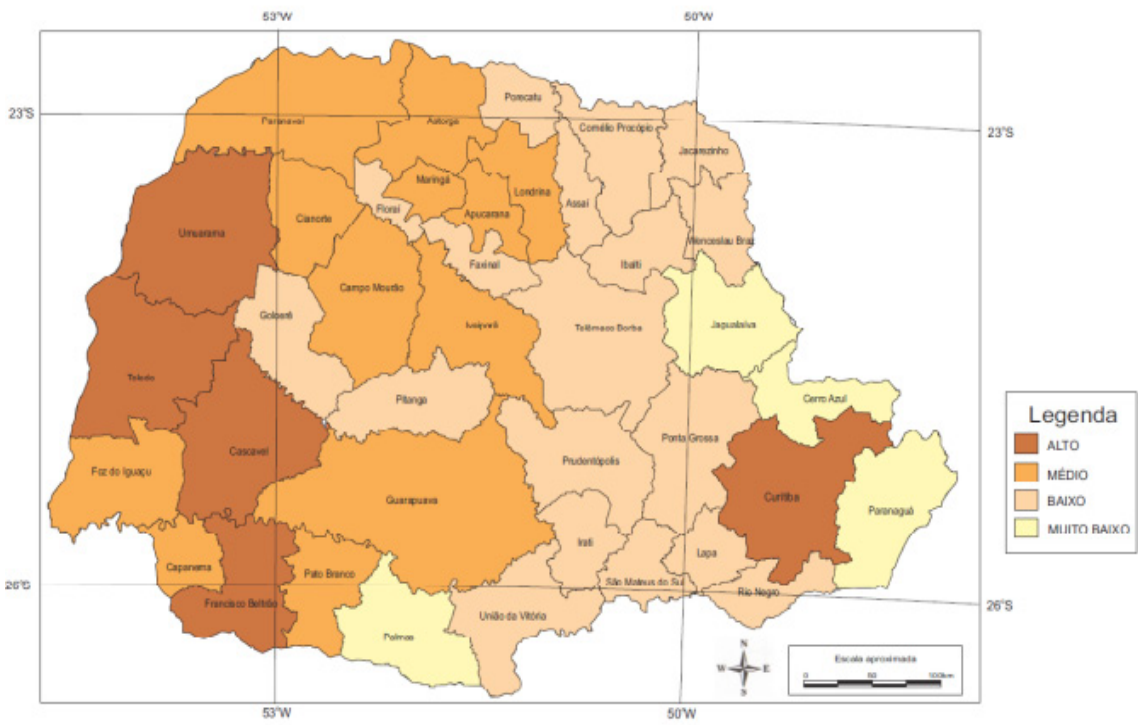

Fonte: Resultados da pesquisa.

\section{Considerações Finais}

A pesquisa alentada sobre a questão do desenvolvimento merece atenção específica, justamente por se tratar de um conteúdo complexo. As definições sobre desenvolvimento regional induzem o pesquisador definir variáveis fundamentadas que traduzem este fenômeno. Neste sentido, buscou-se aferir uma medida sintética das microrregiões geográficas do estado do Paraná, embasada pelo pressuposto econômico e social, através de uma análise relativa dos fatores, apoiada pelo método da análise fatorial, a extração de três fatores: F1 - expressa o grau de aglomeração de pessoas, as melhores condições da economia, educação e do bem estar das microrregiões; F2 - representatividade do setor rural para a microrregião e; F3 - representa a renda e a qualidade de vida. Estes fatores explicam 87,625\% da variância total do modelo. Em seguida, deu-se a classificação destas microrregiões em ordem decrescente. 
Com exceção de Curitiba, que possui uma melhor infraestrutura por ser uma região metropolitana, para disseminar o desenvolvimento regional que é fortemente associado ao Fator 1 (grau de aglomeração de pessoas, as melhores condições da economia, educação e do bem estar da microrregião), as demais microrregiões que também possuem NDR alto, destaca-se o Fator 2 (representatividade do setor rural) como fator relevante de desenvolvimento local e regional.

Com os resultados extraídos, é possível concluir que a maioria das microrregiões do estado do Paraná concentra-se com nível de desenvolvimento regional igual e/ou inferior à média, expressado $87,18 \%$ do total da microrregiões, 0 que se torna evidente a necessidade de maior atenção para as regiões que exibiram tal resultado. A análise dos escores fatoriais de cada região pode apontar para as particularidades de cada uma, ou seja, os aspectos que em cada uma merece maior vigilância, no sentido de melhorar a sua condição e desenvolvimento regional.

Portanto, os resultados alcançados neste estudo não são pretextos de extinguir pesquisas de aprofundamento sobre o tema, pois o aperfeiçoamento do assunto bem como a pesquisa da realidade regional possibilitam o fornecimento de informações que permitem apontar possíveis desigualdades sociais e econômicas. Além disso, o aprimoramento de pesquisas também favorece a eficiência da intervenção estatal por meio de políticas públicas direcionadas a alcançar uma similaridade do nível de desenvolvimento regional em todas as microrregiões do estado do Paraná.

\section{Referências}

Aydalot, P. (1985). Economie régionale et urbaine. Paris: Economica.

Barroso, L.P.; Artes, R (2003). Análise multivariada. 48a Reunião Anual da Região Brasileira da Sociedade Internacional de Biometria e 100 Simpósio de Estatística Aplicada à Experimentação Agronômica. Lavras, 2003.

Ferreira Júnior, S. et al. (2004). A Modernização Agropecuária nas Microrregiões do Estado de Minas Gerais. RER, Rio de Janeiro, vol. 42(1), pp. 73-89.

Ferreira Júnior, S.; Baptista, A J.M.S. E Lima, J. E. (2003). “A modernização agropecuária nas microrregiões do estado de Minas Gerais". Congresso da SOBER. SOBER. Juiz de Fora.

Haddad, P.R. et al. (1989). Economia regional. Fortaleza, BNB.

Hair, J. F.; Anderson, R. E.; Tatham, R. L.; Black, W. C. (1998). Multivariate data analysis. 4 ed. New Jersey: Prentice Hall.

Hirschman, A. O (1958). The strategy of economic development. New Haven: Yali 
University Press, 1958.

Hirschman, A. O. (1961). Estratégia do Desenvolvimento Econômico. Rio de Janeiro: Fundo de Cultura.

Hoffmann, R. (1999). Componentes principais e análise fatorial. Piracicaba: ESALQ.

Ibge - Instituto Brasileiro De Geografia E Estatística (2013). Censo Demográfico 2010. Disponível em: <http://www.ibge.gov.br/home/estatistica/populacao/ censo2010/default.shtm>. Acesso em: 08 Jun., 2013.

Instituto Paranaense de Desenvolvimento Econômico e Social (IPARDES). URL [on-line]:< http://www.ipardes.gov.br > Acesso em: 05 Jun. 2013.

Mata, H.T.C; Ponciano, N. J.; Souza, P.M. E Mira, E. C. (2004). "Padrão e determinantes do desenvolvimento econômico e social dos municípios do estado da Bahia: a dicotomia rural-urbano”. Congresso da SOBER. SOBER. Campo Grande.

Melo, C. O.; E Parré, J. L. (2007). "Índice de desenvolvimento rural dos municípios paranaenses: determinantes e hierarquização”. Rev. Econ. Sociol. Rural, v.45 (2), pp. 329-365.

Myrdal, G. (1957). Economic Theory and Under-developed Regions. London: Duckworth.

Monteiro, V.P.; Pinheiro, J. C. (2004). “Critério para implantação de tecnologias de suprimentos de água potável em municípios cearenses afetados pelo alto teor de sal”. Revista de Economia e Sociologia Rura,. v. 42 (2) pp. 365-387.

Perroux, F. (1955). "Note sur la Notion de Pôle de Croissance”. Économie Appliquée, v. 7, pp. 307-320, Paris.

Rezende, L.P. E Parré, J. L. (2003b). “Comparação do grau de desenvolvimento agrícola dos municípios paranaenses”. Congresso da SOBER. Juiz de Fora.

Rezende, L.P. E Parré, J. L. (2004). “A regionalização da agricultura paranaense na década de noventa: um estudo utilizando estatística multivariada”. Congresso da SOBER, SOBER.

Rosado, P.L; Rossato, M.V. E Lima, J. E. (2005). "Hierarquização e desenvolvimento sócio-econômico das microrregiões de Minas Gerais: uma análise regional”. Congresso da SOBER, Ribeirão Preto.

Secretaria de Estado da Agricultura e do Abastecimento - SEAB, Departamento de Economia Rural - DERAL; Disponível em: < http://www.seab.pr.gov.br > Acesso em: 06 Jun., 2013.

Sorgi, F. A. (2009). Desenvolvimento Econômico Regional: Um Estudo do Norte Pioneiro do Paraná - Microrregião de Cornélio Procópio, UFPR.

Stege, A. L. ; Parre, J. L. (2011). Desenvolvimento Rural nas Microrregiões do Brasil: Um Estudo Multidimensional (Apresentação de Trabalho/Congresso).

Tavares, H. M. (2010). “Estratégias de desenvolvimento regional. Da grande indústria ao Arranjo Produtivo Local?” Revista Brasileira e Gestão e Desenvolvimento Regional, v. 7, pp. 51 a 68.

Turra, S. E Melo, C. O. (2012). “Desenvolvimento Rural das microrregiões geográficas do estado do Paraná”. IV Simpósio Brasileiro de Agropecuária Sustentável e I congresso Internacional de Agropecuária Sustentável, Porto Alegre. 
Udernan, S. (2007). "Políticas de Desenvolvimento Regional no Brasil: Circunscrevendo o Potencial das Novas Iniciativas”. XXXI Encontro da Anpad. Rio de Janeiro.

Udernan, S. (2008). "Políticas de Desenvolvimento Regional no Brasil: Limites de uma nova agenda para Nordeste”. Revista: Associação Brasileira de Estudos Regionais e Urbanos.

Xerxenevsky, L. L. E Fochezatto, A. (2011). “Índice Relativo de Desenvolvimento Socioeconômico dos Municípios do Litoral do Rio Grande do Sul: uma aplicação da análise fatorial». XIV ANPEC SUL, Florianópolis.

Zambrano, C.E. E Pinto, W. J. (2004). “Avaliação do desenvolvimento municipal do estado de Mato Grosso aplicando análise multivariada”. Congresso da SOBER. SOBER, Campo Grande. 